\title{
BOSONIZATION OF THE SCHWINGER MODEL BY NONCOMMUTATIVE CHIRAL BOSONS
}

\author{
J. BEN GELOUN,$^{\dagger}$ J. GOVAERTS ${ }^{\dagger, \ddagger}$ and M. N. HOUNKONNOU ${ }^{\dagger}$ \\ ${ }^{\dagger}$ International Chair in Mathematical Physics and Applications (ICMPA), \\ 072 B.P. 50, Cotonou, Republic of Benin \\ E-mail: jobengeloun@yahoo.fr,norbert_hounkonnou@cipma.net \\ ¥Center for Particle Physics and Phenomenology (CP3), \\ Institute of Nuclear Physics, Catholic University of Louvain, \\ 2, Chemin du Cyclotron, B-1348 Louvain-la-Neuve, Belgium \\ E-mail: Jan.Govaerts@fynu.ucl.ac.be
}

\begin{abstract}
Bosonization of the Schwinger model with noncommutative chiral bosons is considered on a spacetime of cylinder topology. Using point splitting regularization, manifest gauge invariance is maintained throughout. Physical consequences are discussed.
\end{abstract}

To appear in the Proceedings of the Fourth International Workshop on Contemporary Problems in Mathematical Physics, November $5^{\text {th }}-11^{\text {th }}, 2005$, Cotonou (Republic of Benin), eds. J. Govaerts, M. N. Hounkonnou and A. Z. Msezane

(World Scientific, Singapore, 2006).

CP3-06-09

ICMPA-MPA/2006/26 


\section{Introduction}

Bosonization $^{1}$ proves to be a successful method for quantization in noncommutative field theory. ${ }^{2-5}$ On the other hand, it is well known that the ordinary massless Schwinger model is exactly soluble through the bosonization of the massless fermion, in particular within the physical projector method which avoids having to perform any gauge fixing procedure. ${ }^{6}$ In this contribution, we investigate a new quantized version of the Schwinger model via a noncommutative chiral bosonization, taking the spacelike dimension to be compactified into a circle, $\mathbb{R} \rightarrow S^{1}$. The quantization to be considered presently is realized in a noncommutative field space instead of a noncommutative spacetime. The Hamiltonian analysis is significantly simplified without loss of a meaningful physical interpretation. The quantized system thereby obtained generalizes the nonperturbative quantization of the model $^{6}$ as an asymptotic $\theta$-quantization, and the quantization rules found by Das et al. ${ }^{2}$ are extended to a nontrivial spacetime topology. Furthermore, we provide a gauge invariant quantized model by the point splitting regularization method using the Wilson line phase factor. The Hamiltonian operator is diagonalized leading to a successful nonperturbative quantization. Such an abelian gauge theory based on the circle $S^{1}$ as the space topology is new to the best of our knowledge.

Section 2 presents our notations and briefly describes the classical Schwinger model and the relevant quantities of the constrained dynamics. In Sec. 3, we study chiral bosons in the noncommutative field space and develop the nonperturbative quantization of the model based on the bosonization of the fermionic degrees of freedom. The gauge invariant regularization is given in Sec. 4. Finally, some conclusions are presented in Sec. 5 .

\section{The Classical Schwinger Model}

Henceforth, the topology of the $1+1$ dimensional spacetime is that of the cylinder $\mathbb{R} \times S^{1}$, where $\mathbb{R}$ stands for the timelike coordinate and the torus $S^{1}$ of radius $R$ and circumference $L=2 \pi R$ for the spacelike one. The Minkowski metric is $\eta_{\mu \nu}=\operatorname{diag}(+,-), \mu, \nu=0,1$. Units such that $\hbar=$ $1=c$ are used throughout. The antisymmetric tensor $\epsilon^{\mu \nu}$ is such that $\epsilon^{01}=1=-\epsilon^{10}$. In the chiral representation, the Clifford-Dirac algebra $\left\{\gamma^{\mu}, \gamma^{\nu}\right\}=2 \eta^{\mu \nu}$ is given by $\gamma^{0}=\sigma^{1}, \gamma^{1}=i \sigma^{2}$ and $\gamma_{5}=\gamma^{0} \gamma^{1}=-\sigma^{3}$, where $\sigma^{i}(i=1,2,3)$ are the usual Pauli matrices. 
4

The field degrees of freedom of the model are the real $U(1)$ gauge vector field $A_{\mu}\left(x^{\mu}\right)$ and a single massless Dirac spinor $\psi\left(x^{\mu}\right)$ represented by Grassmann odd variables, describing the fermionic particles. The Dirac spinor decomposes into two complex Weyl spinor representations of opposite chiralities, $\psi(t, x)=\psi_{+}(t+x)+\psi_{-}(t-x)$, such that $\gamma_{5} \psi_{ \pm}=\mp \psi_{ \pm}$. Furthermore, the following choice of periodic and twisted (or anti-periodic) boundary conditions on the circle is assumed, respectively,

$$
A_{\mu}(t, x+L)=A_{\mu}(t, x), \quad \psi_{ \pm}(t, x+L)=-e^{2 i \pi \alpha_{ \pm}} \psi_{ \pm}(t, x),
$$

$\alpha_{ \pm}$being arbitrary real parameters defined modulo any integer such that $\alpha_{+}=\alpha_{-}=\alpha(\bmod \mathbb{Z})$ in order to ensure parity invariance.

The model is described by the Lagrangian density given in a Lorentz and gauge invariant form as (Einstein's summation convention is implicit)

$$
\mathcal{L}=-\frac{1}{4} F_{\mu \nu} F^{\mu \nu}+\frac{1}{2} i \bar{\psi} \gamma^{\mu}\left(\partial_{\mu}+i e A_{\mu}\right) \psi-\frac{1}{2} i\left(\partial_{\mu}-i e A_{\mu}\right) \bar{\psi} \gamma^{\mu} \psi
$$

with $F_{\mu \nu}=\partial_{\mu} A_{\nu}-\partial_{\nu} A_{\mu}$, the gauge field strength corresponding, in $1+1$ dimensions, to a single field $F_{01}$ which is the pseudo-scalar electric field $E$; $e$ stands for the gauge coupling constant which, up to a sign, is the charge of the fermionic particle (electron or positron).

Gauge invariance of the system implies a constrained dynamics ${ }^{6}$ whose Dirac Hamiltonian treatment leads to a first-class constraint $\Phi$ (Gauss' law) and the first-class Hamiltonian density $\mathcal{H}$ given as follows,

$$
\begin{aligned}
\Phi & =\partial_{1} \pi_{1}+e \psi^{\dagger} \psi, \pi_{1}=-E, \\
\mathcal{H} & =\frac{1}{2} \pi_{1}^{2}-\frac{1}{2} i \psi^{\dagger} \gamma_{5}\left(\partial_{1}-i e A^{1}\right) \psi+\frac{1}{2} i\left(\partial_{1}+i e A^{1}\right) \psi^{\dagger} \gamma_{5} \psi,
\end{aligned}
$$

where $\pi_{1}$ is the momentum conjugate to $A^{1}$. In this system, $A^{0}$ turns out to be a Lagrange multiplier for $\Phi$. The fundamental graded Dirac-Poisson algebra, taken at equal time, reads $(a, b=+,-)$,

$$
\left\{A^{1}(t, x), \pi_{1}(t, y)\right\}=\delta(x-y), \quad\left\{\psi_{a}(t, x), \psi_{b}^{\dagger}(t, y)\right\}=-i \delta_{a b} \delta(x-y) .
$$

\section{Nonperturbative Deformed Quantization}

Bosonization of the fermionic sector of the Schwinger model involves the quantum deformed algebra of chiral bosons, $\phi_{+}(t, x)$ and $\phi_{-}(t, x)$. In the Schrödinger picture at time $t=0$, we have the Fourier mode decomposition of the chiral fields $\phi_{ \pm}$, 


$$
\begin{aligned}
\phi_{ \pm}(x)= & \frac{1}{\sqrt{4 \pi}}\left\{q_{ \pm}+\frac{2 \pi}{L} p_{ \pm}( \pm x)\right. \\
& \left.+\sum_{n=1}^{+\infty} \frac{1}{\sqrt{n}}\left[\mathbf{a}_{ \pm, n} e^{-\frac{2 i \pi}{L} n( \pm x)}+\mathbf{a}_{ \pm, n}^{\dagger} e^{\frac{2 i \pi}{L} n( \pm x)}\right]\right\} .
\end{aligned}
$$

Representing a noncommutative field space, the deformed Dirac algebra of the chiral bosons proposed by Das et al. ${ }^{2}$ induces the following quantum mode algebras corresponding to the chiral fields $\phi_{ \pm}$,

$$
\begin{aligned}
{\left[q_{a}, p_{b}\right] } & =i \Delta_{a b}, \quad\left[\mathbf{a}_{a, n}, \mathbf{a}_{b, m}^{\dagger}\right]=\delta_{a b} \delta_{n, m}, \\
{\left[\mathbf{a}_{a, n}, \mathbf{a}_{b, m}\right] } & =-\epsilon_{a b} \theta \delta_{n, m}=-\left[\mathbf{a}_{a, n}^{\dagger}, \mathbf{a}_{b, m}^{\dagger}\right],
\end{aligned}
$$

with $a, b=+,-, \theta$ a real parameter, and $\Delta_{a b}=\epsilon_{a b} \theta+\delta_{a b}$. Furthermore, we assume the hermiticity conditions for the zero modes $q_{a}=q_{a}^{\dagger}$ and $p_{a}=p_{a}^{\dagger}$.

In terms of these bosonic modes, the fermionic operator $\psi_{a}$ is written as $\psi_{a}(z)=F(z) V_{a}(z)$ with $V_{a}$ the vertex operator defined as

$$
V_{a}=K(a): e^{a i \beta\left(\varphi_{a}-a \theta \varphi_{-a}\right)}:,
$$

where $F(z)$ is a holomorphic function, $\varphi_{a}=\sqrt{4 \pi} \phi_{a}$ and $K(a)$ is fixed to $K(a)=e^{\frac{i \pi}{2} \rho_{a} p_{-a}}$. The constant factors $\beta$ and $\rho_{a}$ are determined by the bosonization process. The vertex operators $V_{a}$ and $V_{a}^{\dagger}$ are written as Laurent series as

$$
\begin{gathered}
V_{ \pm}(z)=\sum_{n=-\infty}^{\infty} \psi_{ \pm, n} z^{-\left(n \mp \frac{1}{2} \mp \alpha_{ \pm}\right)-\frac{1}{2}} \\
V_{ \pm}^{\dagger}(z)=\sum_{n=-\infty}^{\infty} \psi_{ \pm, n}^{\dagger} z^{\left(n \mp \frac{1}{2} \mp \alpha_{ \pm}\right)-\frac{1}{2}}
\end{gathered}
$$

From (8) and (9), we deduce, by a complex contour integral around the origin $z=0$, the expressions for the mode operators $\psi_{ \pm, n}$ and $\psi_{ \pm, n}^{\dagger}$ as

$\psi_{ \pm, n}=\oint_{C} \frac{d z}{2 i \pi} z^{\left(n \mp \frac{1}{2} \mp \alpha_{ \pm}\right)-\frac{1}{2}} V_{ \pm}(z), \quad \psi_{ \pm, n}^{\dagger}=\oint_{C} \frac{d z}{2 i \pi} z^{-\left(n \mp \frac{1}{2} \mp \alpha_{ \pm}\right)-\frac{1}{2}} V_{ \pm}^{\dagger}(z)$.

By well established conformal field theory techniques, ${ }^{7}$ the fermionic mode anticommutators are evaluated. One has,

$$
\begin{aligned}
{\left[\psi_{a, n}, \psi_{b, m}^{\dagger}\right]_{+} } & =\oint_{C} \frac{d z}{2 i \pi} \oint_{C} \frac{d \omega}{2 i \pi} z^{N-\frac{1}{2}} \omega^{-M-\frac{1}{2}}\left[V_{a}(z), V_{b}^{\dagger}(\omega)\right]_{+} \\
& =\oint_{C} \frac{d z}{2 i \pi} z^{N-\frac{1}{2}} \oint_{z} \frac{d \omega}{2 i \pi} \omega^{-M-\frac{1}{2}} \times R\left(V_{a}(z) V_{b}^{\dagger}(\omega)\right),
\end{aligned}
$$


with $a, b=+,-, N=n-a\left(\frac{1}{2}+\alpha_{a}\right)$ and $M=m-b\left(\frac{1}{2}+\alpha_{b}\right)$. We have used the Wick theorem in (10). Integration over $\omega$ stands for an integration depending on $z$, while the last integral over $z$ is taken around the origin, $z=0$. Using : $e^{A}:: e^{B}:=e^{<A B>}: e^{A} e^{B}:$ where $A, B$ are some operators whose commutator is a $c$-number, we determine the radial ordered product $R\left(V_{a}(z) V_{b}^{\dagger}(\omega)\right)$ as follows,

$$
\begin{aligned}
& R\left(V_{a}(z) V_{b}^{\dagger}(\omega)\right) \\
= & {\left[\Theta(|z|-|\omega|)\left(\frac{1}{(z-\omega)^{\delta_{a b}}} z^{-\theta \epsilon_{a b}}\right)^{\beta^{2}\left(1+\theta^{2}\right)} e^{a \beta \rho_{a} \frac{i \pi}{2}\left(1+\theta^{2}\right) \delta_{a(-b)}}\right.} \\
& \left.-\Theta(|\omega|-|z|)\left(\frac{1}{(\omega-z)^{\delta_{a b}}} \omega^{\theta \epsilon_{a b}}\right)^{\beta^{2}\left(1+\theta^{2}\right)} e^{-a \beta \rho_{-a} \frac{i \pi}{2}\left(1+\theta^{2}\right) \delta_{a(-b)}}\right] \\
& \times: e^{i \frac{\pi}{2}\left(\rho_{a} p_{-a}-\rho_{b} p_{-b}\right)+i \beta\left(a\left(\varphi_{a}-a \theta \varphi_{-a}\right)(z)-b\left(\varphi_{b}-b \theta \varphi_{-b}\right)(\omega)\right)}:,
\end{aligned}
$$

where $\Theta(\cdot)$ denotes the Heaviside step function. The different parameters are fixed by

$$
\beta= \pm \lambda\left(1+\theta^{2}\right)^{-\frac{1}{2}}, \quad \rho_{a}=\rho_{-a}=\rho\left(1+\theta^{2}\right)^{-\frac{1}{2}}, \quad \text { with } \lambda^{2}=1=\rho^{2} .
$$

The induced singular part of the short distance operator, as $z \rightarrow \omega$, reads

$$
R\left(V_{a}(z) V_{a}^{\dagger}(\omega)\right)=\frac{1}{(z-\omega)}+\cdots
$$

so that, by the residue theorem and (13), we get

$$
\left[\psi_{a, n}, \psi_{b, n}^{\dagger}\right]_{+}=\delta_{a b} \delta_{n m}
$$

which reproduces the well known anticommutator of the corresponding fermionic operators. In the same manner, given (12), evaluating the other radial ordered products $R\left(V_{a}^{\dagger}(z) V_{b}(\omega)\right), R\left(V_{a}(z) V_{b}(\omega)\right)$ and $R\left(V_{a}^{\dagger}(z) V_{b}^{\dagger}(\omega)\right)$ and then integrating the corresponding fermionic algebra, the remaining fermionic mode anticommutators are recovered as follows,

$$
\left[\psi_{a, n}^{\dagger}, \psi_{b, n}\right]_{+}=\delta_{a b} \delta_{n m}, \quad\left[\psi_{a, n}, \psi_{b, n}\right]_{+}=0, \quad\left[\psi_{a, n}^{\dagger}, \psi_{b, n}^{\dagger}\right]_{+}=0
$$

Indeed, as $z \rightarrow \omega$, the only singularity such as the one in (13) with a nonvanishing residue occurs for $R\left(V_{a}^{\dagger}(z) V_{b}(\omega)\right)$. We complete this study by giving the bosonized fermionic field (the $\theta$-Mattis-Mandelstam formula), 


$$
\begin{aligned}
\psi_{ \pm}(x)= & \frac{1}{\sqrt{L}} e^{ \pm \frac{i \pi}{L} x} e^{\frac{ \pm i \lambda}{\sqrt{1+\theta^{2}}}\left(q_{ \pm} \mp \theta q_{\mp}\right)} e^{\frac{i \pi}{2}} \frac{\rho p_{\mp}}{\sqrt{1+\theta^{2}}} e^{\frac{2 i \pi \lambda}{L \sqrt{1+\theta^{2}}}}\left(p_{ \pm} \pm \theta p_{\mp}\right) x \\
& \times \prod_{n=1}^{\infty} e^{\frac{ \pm i \lambda}{\sqrt{n} \sqrt{1+\theta^{2}}}\left(\mathbf{a}_{ \pm, n}^{\dagger} e^{ \pm \frac{2 i \pi}{L} n x} \mp \theta \mathbf{a}_{\mp, n}^{\dagger} e^{\mp \frac{2 i \pi}{L} n x}\right)} \\
& \times \prod_{n=1}^{\infty} e^{\frac{ \pm i \lambda}{\sqrt{n} \sqrt{1+\theta^{2}}}\left(\mathbf{a}_{ \pm, n} e^{\mp \frac{2 i \pi}{L} n x} \mp \theta \mathbf{a}_{\mp, n} e^{ \pm \frac{2 i \pi}{L} n x}\right)}
\end{aligned}
$$

The holonomy boundary condition (h.b.c.) of the chiral bosons should also be related to $\alpha_{ \pm}$, namely the h.b.c. of the fermionic operator $\psi_{ \pm}$, through the deformed Heisenberg algebra on the circle. This relation has still to be understood (this work is in progress). Finally, the bosonic fields $\phi_{ \pm}$and their quantum states also provide a representation of the fermionic algebra irrespective of the choices $\rho= \pm 1$ and $\lambda= \pm 1$.

\section{Gauge Invariant Regularization}

The first-class quantities (3) and (4) are composite operators which require a choice of operator ordering which should remain anomaly free and gauge invariant. Since gauge invariance must be preserved at all steps, a gauge invariant point splitting regularization of short distance singularities is a relevant choice. ${ }^{6}$ For instance, instead of the products $\psi_{ \pm}^{\dagger}(x) \psi_{ \pm}(x)$, one considers the gauge invariant quantities

$$
\lim _{y \rightarrow x} \psi_{ \pm}^{\dagger}(y) e^{i e \int_{x}^{y} d u A^{1}(u)} \psi_{ \pm}(x)
$$

and subtracts the divergent terms. Through this procedure, the fermionic currents in the bosonized representation are given by

$$
\psi_{ \pm}^{\dagger} \psi_{ \pm}=-\frac{\lambda}{2 \pi \sqrt{1+\theta^{2}}}\left[\partial_{1}\left(\varphi_{ \pm} \mp \theta \varphi_{\mp}\right) \mp \lambda e \sqrt{1+\theta^{2}} A^{1}\right],
$$

so that the vector and axial gauge invariant currents are expressed as, respectively,

$$
\begin{aligned}
\psi^{\dagger} \psi & =-\frac{\lambda}{2 \pi \sqrt{1+\theta^{2}}}\left[\partial_{1}\left(\varphi_{+}+\varphi_{-}\right)+\theta \partial_{1}\left(\varphi_{+}-\varphi_{-}\right)\right], \\
\psi^{\dagger} \gamma_{5} \psi & =-\frac{e}{\pi}\left[A^{1}-\frac{1}{2 \lambda e \sqrt{1+\theta^{2}}}\left[\partial_{1}\left(\varphi_{+}-\varphi_{-}\right)-\theta \partial_{1}\left(\varphi_{+}+\varphi_{-}\right)\right]\right] .
\end{aligned}
$$

Denoting the regularized current components by $J^{ \pm}=\psi_{ \pm}^{\dagger} \psi_{ \pm}$, the two coupled $U(1)$ Kac-Moody algebras ${ }^{2}\left[J^{a}, J^{b}\right]=\left(a \delta_{a b}+\epsilon_{a b} \theta\right) \frac{i}{2} \partial_{x} \delta(x-y)$ for the unmixed and mixed commutators are still valid. The gauge contribution 
is indeed null. Furthermore, we have the regularized vector and axial charges given by

$$
\begin{aligned}
Q & =-\frac{\lambda}{\sqrt{1+\theta^{2}}}\left[p_{+}-p_{-}+\theta\left(p_{+}+p_{-}\right)\right] \\
Q_{5} & =-\frac{e}{\pi} \int_{0}^{L} d x A^{1}+\frac{\lambda}{\sqrt{1+\theta^{2}}}\left[p_{+}+p_{-}-\theta\left(p_{+}-p_{-}\right)\right] .
\end{aligned}
$$

One notices that the quantum axial anomaly of the axial charge, due to the zero mode of the gauge potential $A^{1}$, remains as in the commutative case. The regularized first-class constraint reads

$$
\Phi=\partial_{1} \pi_{1}-\frac{e \lambda}{2 \pi \sqrt{1+\theta^{2}}}\left[\partial_{1}\left(\varphi_{+}+\varphi_{-}\right)+\theta \partial_{1}\left(\varphi_{+}-\varphi_{-}\right)\right] .
$$

The bilinears involving the covariant derivatives, namely, $\psi_{ \pm}^{\dagger} D_{1} \psi_{ \pm}$and $D_{1} \psi_{ \pm}^{\dagger} \psi_{ \pm}$, are also regularized by the point splitting method. Setting $D_{1} \psi=\left(\partial_{1}-i e A^{1}\right) \psi$ and $D_{1} \psi^{\dagger}=\left(\partial_{1}+i e A^{1}\right) \psi^{\dagger}$, we obtain the fermionic contributions to the first-class Hamiltonian

$$
\begin{aligned}
H_{ \pm} & =\frac{i}{2} \psi_{ \pm}^{\dagger} D_{1} \psi_{ \pm}-\frac{i}{2} D_{1} \psi_{ \pm}^{\dagger} \psi_{ \pm} \\
& = \pm \frac{1}{4 \pi\left(1+\theta^{2}\right)}\left(\partial_{1}\left(\varphi_{ \pm} \mp \theta \varphi_{\mp}\right) \mp \lambda e \sqrt{1+\theta^{2}} A^{1}\right)^{2} \mp \frac{\pi}{12 L^{2}},
\end{aligned}
$$

showing that the deformation has not modified the Casimir energy $\pi /\left(12 L^{2}\right)$.

The first-class gauge invariant Hamiltonian density reads

$$
\begin{aligned}
\mathcal{H}= & \frac{1}{2} \pi_{1}^{2}+\frac{1}{4 \pi\left(1+\theta^{2}\right)}\left[\left(\partial_{1}\left(\varphi_{+}-\theta \varphi_{-}\right)-\lambda e \sqrt{1+\theta^{2}} A^{1}\right)^{2}\right. \\
& \left.+\left(\partial_{1}\left(\varphi_{-}+\theta \varphi_{+}\right)+\lambda e \sqrt{1+\theta^{2}} A^{1}\right)^{2}\right] .
\end{aligned}
$$

Introducing the change of variables

$$
\begin{aligned}
\Upsilon & =\frac{-1}{\mu} \pi_{1}, \\
\pi_{\Upsilon} & =\mu\left\{\left(A^{1}-\frac{1}{2 e \lambda \sqrt{1+\theta^{2}}} \partial_{1}\left[\left(\varphi_{+}-\varphi_{-}\right)-\theta\left(\varphi_{+}+\varphi_{-}\right)\right]\right\},\right.
\end{aligned}
$$

with $\mu=|e| / \sqrt{\pi}$, the first-class Hamiltonian density (21) can be expressed using the new degrees of freedom $\left(\Upsilon, \pi_{\Upsilon}, \varphi_{a}, \partial_{1} \varphi_{a}\right)$ as

$$
\mathcal{H}=\frac{1}{2} \pi_{\Upsilon}^{2}+\frac{1}{2}\left(\partial_{1} \Upsilon\right)^{2}+\frac{1}{2} \mu^{2} \Upsilon^{2}+\frac{1}{2}\left(\frac{\Phi}{\mu}\right)^{2}+\left(\frac{\Phi}{\mu}\right) \partial_{1} \Upsilon
$$


Finally, given $\Delta_{a b}=\epsilon_{a b} \theta+\delta_{a b}$, we have the bosonic algebra

$$
\begin{aligned}
& {\left[\Upsilon(x), \pi_{\Upsilon}(y)\right]=i \delta(x-y), \quad\left[\varphi_{a}, \partial_{1} \varphi_{b}\right]=2 i b \pi \Delta_{a b} \delta(x-y),} \\
& {\left[\varphi_{a}, \pi_{\Upsilon}(y)\right]=-\frac{i \pi \mu}{e \lambda} \sqrt{1+\theta^{2}} \delta(x-y) .}
\end{aligned}
$$

\section{Concluding Remarks}

In this work, we have generalized to the cylinder spacetime topology $\mathbb{R} \times S^{1}$ and to the massless Schwinger model the noncommutative bosonization induced by a deformed Dirac algebra of noncommutative chiral bosons. The usual quantum theory is recovered as $\theta \rightarrow 0$. This quantum model, regularized by the gauge invariant point splitting method, induces a gauge invariant dynamics equivalent to that of an electric field of mass $\mu=|e| / \sqrt{\pi}$, like in the ordinary commutative case. Furthermore, due to the choice of the chiral boson algebra, a charge rescaling $e \rightarrow e \sqrt{1+\theta^{2}}$ appears in the expressions. This fact has still to be related to the equivalence found by Das et al. ${ }^{2}$ which states that noncommutative chiral bosons are equivalent to free fermionic fields moving with a speed equal to $c^{\prime}=c \sqrt{1+\theta^{2}}, c$ being the speed of light in vacuum. We expect that, in a forthcoming investigation, a stronger deformation involving the nonzero mode algebra of the chiral fields would lead to a noncommutative spinor field theory.

\section{Acknowledgments}

J. B. G. is grateful to the Abdus Salam International Centre for Theoretical Physics (ICTP, Trieste, Italy) for a Ph.D. fellowship under the grant Prj-15. J. G. acknowledges the Abdus Salam ICTP Visiting Scholar Programme in support of a Visiting Professorship at the International Chair in Mathematical Physics and Applications (ICMPA). The work of J. G. is partially supported by the Belgian Federal Office for Scientific, Technical and Cultural Affairs through the Interuniversity Attraction Pole (IAP) $\mathrm{P} 5 / 27$.

\section{References}

1. The literature on bosonization is extensive, see for instance, D. Mattis, J. Math. Phys. 15, 609 (1974);

S. Coleman, Phys. Rev. D 11, 2088 (1975).

2. A. Das, J. Gamboa, F. Mendez and J. Lopez-Sarrion, JHEP 05, 022 (2004).

3. S. Gosh, Phys. Lett. B 563, 112 (2003).

4. O. Lechtenfeld, L. Mazzanti, S. Penati and L. Tamassia, Nucl. Phys. B 705, 477 (2005). 
5. For a rewiew on Noncommutative Field Theory, see,

M. R. Douglas and N. A. Nekrasov, Rev. Mod. Phys. 73, 977 (2001).

6. G. Y. H. Avossevou and J. Govaerts, Proceedings of the Second International Workshop on Contemporary Problems in Mathematical Physics, Cotonou, Benin, eds. J. Govaerts, M. N. Hounkonnou and A. Z. Mzesane (World Scientific, Singapore, 2002), pp. 374-394.

7. P. Ginsparg, Applied Conformal Field Theory, Lectures at the Les Houches Summer Session, June 28-August 5, 1988, in Fields, Strings and Critical Phenomena (Les Houches, Session XLIX, 1988), eds. E. Brézin and J. Zinn Justin (North Holland, Amsterdam, 1990); e-preprint arXiv:hep-th/9108028. 